\title{
SOME PHYSICAL MEASUREMENTS IN THE FIRST THREE YEARS OF LIFE
}

\author{
BY \\ FRANK FALKNER* \\ From the Child Study Centre, Institutes of Child Health and Education, University of London
}

(RECEIVED FOR PUBLICATION JULY 1, 1957)

There are few standards of physical measurements available for pre-school children in the British Isles. Gore and Palmer (1949) published cross-sectional data of the pre-school period based on measurements taken in 1947. Within each age-group sample there were differences of weeks or months between ages of individual children. The most recent publication of cross-sectional data in this age period was from the Oxford Child Health Survey in 1955 (Acheson, Kemp and Parfit, 1955) and gives standards of height and weight for children born between 1944 and 1948. This was a longitudinal study, but no incremental data were given. The late Professor Low's (1952) raw data have been published on several measurements for a group of children followed for the first five years of life and who were born between 1923 and 1927. So far as is known these are the only longitudinal data in this age period in the United Kingdom published up till now.

The following standards of children born between 1952 and 1954 are presented. For details of the sample, method of recruitment and investigations carried out, etc., reference may be made to Moore, Hindley and Falkner (1954). Briefly, the sample consists of 'healthy' unselected children, and these same children are followed regularly from birth. They are drawn from an area in London. The children are seen at the ages of 4 weeks ( \pm 2 days), $13,26,39$ weeks, 1 year, 18 months ( \pm 7 days), 2 years and thence yearly ( \pm 14 days). The majority of children are seen very close to the correct date and never outside these stated limits.

It may be remarked that at 3 years the number of children forming the sample drops considerably. At the time of analysis not all the original children had reached 3 years of age - a common happening in running analyses of longitudinal studies.

\footnotetext{
* Present address: University of Louisville Medical School, Louisville, Kentucky.
}

\section{Measurements}

All the measurements were taken by one of four measurers, the majority being taken by one person, and the rest divided equally between the other three. All were trained. When a new measurer joined the team he or she was taught by a trained member, and checks of individual errors and variations were made from time to time. Lincoln (1930) has shown the very great improvement in accuracy after such training whereby important large errors are reduced to minimal ones.

\section{Increments}

Known gains in specific measurements are a valuable indication of rate of growth and progress. Krogman (1956) has said: 'It is a source of great weakness that this aspect of growth analysis is so consistently neglected.' Increments may be calculated only from children followed longitudinally and not from cross-sectional data. Increments presented in this paper are means calculated on children seen at the beginning and end of an incremental period. (Not all the children are able to present themselves for their appointed examinations every time.) 'Adjusted mean increments' will be given and the term needs explanation. It was clearly convenient to use 13 weeks as the standard comparative incremental period. Not all the intervals are 13 weeks; for example, 4 to 13 weeks is 9 weeks. These intervals were therefore 'adjusted' to bring them to a comparable 13-week period. This may not be entirely permissible, for it presumes that the rate of growth of the particular measurement is rectilinear at that time which may not, in fact, be so; but the periods between examinations are felt to be short enough to justify this where it has been necessary.

In presenting the increments of the children it might be argued that those children who were able to be included by virtue of being seen at the beginning and end of each incremental period, and 
TABLE 1

MEAN RECUMBENT-LENGTH OF CHILD STUDY CENTRE CHILDREN AT 4 WEEKS TO 3 YEARS (In inches to nearest 0.1 inch, and $\mathrm{cm}$. to nearest $\mathrm{mm}$. $1 \mathrm{~S} . \mathrm{D}$. of mean given)

\begin{tabular}{|c|c|c|c|c|c|c|c|c|c|c|c|c|}
\hline & & & \multicolumn{5}{|c|}{ Boys } & \multicolumn{5}{|c|}{ Girls } \\
\hline \multicolumn{3}{|c|}{ Ages } & \multirow{2}{*}{ No. } & \multicolumn{2}{|c|}{ Inches } & \multicolumn{2}{|c|}{$\mathrm{Cm}$} & \multirow{2}{*}{ No. } & \multicolumn{2}{|c|}{ Inches } & \multicolumn{2}{|c|}{$\mathrm{Cm}$} \\
\hline & & & & Mean & S.D. & Mean & S.D. & & Mean & S.D. & Mean & S.D. \\
\hline $\begin{array}{l}4 \text { wks. } \\
13 \text { wks. } \\
26 \text { wks. } \\
39 \text { wks. } \\
1 \text { yr. } \\
18 \text { mos. } \\
2 \text { yrs. } \\
3 \text { yrs. }\end{array}$ & $\begin{array}{l}\ldots \\
\ldots \\
\ldots \\
\ldots \\
\cdots \\
\ldots\end{array}$ & $\begin{array}{l}\ldots \\
\cdots \\
\ldots \\
\cdots \\
\cdots \\
\ldots\end{array}$ & $\begin{array}{l}82 \\
96 \\
94 \\
84 \\
80 \\
77 \\
55 \\
28\end{array}$ & $\begin{array}{l}21 \cdot 2 \\
23 \cdot 7 \\
26 \cdot 3 \\
28 \cdot 1 \\
29 \cdot 5 \\
32 \cdot 0 \\
34 \cdot 0 \\
37 \cdot 1\end{array}$ & $\begin{array}{l}0.9 \\
0.9 \\
0.9 \\
1.0 \\
1.1 \\
1.2 \\
1.5 \\
1.9\end{array}$ & $\begin{array}{l}53 \cdot 9 \\
60 \cdot 2 \\
66 \cdot 7 \\
71 \cdot 3 \\
75 \cdot 0 \\
81 \cdot 2 \\
86 \cdot 5 \\
94 \cdot 1\end{array}$ & $\begin{array}{l}2 \cdot 3 \\
2 \cdot 4 \\
2 \cdot 4 \\
2 \cdot 6 \\
2 \cdot 7 \\
3 \cdot 2 \\
3 \cdot 7 \\
4 \cdot 8\end{array}$ & $\begin{array}{l}77 \\
85 \\
81 \\
78 \\
78 \\
66 \\
54 \\
21\end{array}$ & $\begin{array}{l}20 \cdot 7 \\
22 \cdot 9 \\
25 \cdot 5 \\
27 \cdot 3 \\
28 \cdot 9 \\
31 \cdot 4 \\
33 \cdot 3 \\
37 \cdot 0\end{array}$ & $\begin{array}{l}0.7 \\
0.6 \\
0.8 \\
1.0 \\
1.0 \\
0.9 \\
1.2 \\
1.4\end{array}$ & $\begin{array}{l}52 \cdot 5 \\
58 \cdot 2 \\
64 \cdot 8 \\
69 \cdot 3 \\
73 \cdot 3 \\
79 \cdot 7 \\
84 \cdot 5 \\
94 \cdot 1\end{array}$ & $\begin{array}{l}1 \cdot 8 \\
1 \cdot 6 \\
2 \cdot 6 \\
2 \cdot 6 \\
2 \cdot 6 \\
2 \cdot 4 \\
3 \cdot 1 \\
3 \cdot 4\end{array}$ \\
\hline
\end{tabular}

thus more regularly, were not necessarily representative of the whole group. With this in mind, this possibility was examined and there was found to be no significant difference between the different groups, at the different ages, in size of recumbentlength, weight, crown-rump measurement or head circumference.

\section{Length and Weight Measurements}

Length. There is a difference in an individual's recumbent-length and standing height. Boyd (1929), on children aged 2-5 years, found differences of 1.0 to $1.5 \mathrm{~cm}$., and with older children differences up to $3.0 \mathrm{~cm}$. occur. Since children who can actually stand are not necessarily able to be stood erect in the correct position (Krogman, 1950), it is felt that measurement of standing height before the age of 3 years and possibly 4 is probably unreliable. All measurements of length in this series of children were taken with the child nude lying on a horizontal measuring table. Such tables have been developed by Todd at Western Reserve University, Thompson at Yale, Schultz at Johns Hopkins University and by Knott (1941), Hindley (1953), Thomson (1956) and Falkner (1957). The technique used is well summarized by Krogman (1950). The estimated maximum error was $\pm 0.5 \%$. Children were measured either in the morning or in the afternoon. Time of day is important in measuring height, for children can 'lose' $2 \mathrm{~cm}$. in a day and $1 \mathrm{~cm}$. can be lost in the first hour. This can be 'put back' again after a rest (Palmer, 1930; Redfield and Meredith, 1938; Boyd, 1929). This source of error is lessened in measuring recumbent-length, but at the same time the children were stretched (not severely) just before actual measurement.

Weight. All children were weighed in the nude to the nearest ounce on either a basket-scale or a platform beam balance (both periodically checked) according to age. Percentiles are given in the weight tables rather than standard deviations, for weight is not normally distributed (it is positively skewed), whereas recumbent-length is nearly so.

Figs. 1 and 2 show graphically* the data from Tables 1, 2, 3 and 4.

* In a study such as this there are several ways of plotting the data (Tanner, 1951), for not all the children are seen every time. Those that are at the beginning and the end of each incremental period only may be used to plot the velocity curve. Hence the distance curve can be plotted by using the maximum number of children seen at each age-in other words, using the longitudinal study crosssectionally - or by plotting it from the velocity curve, having started with a mean size at birth or 4 weeks of age. (These are not the only ways (see Tanner, 1951), but the most simple, practical and least complicated.) All the distance curves plotted in this paper are from means at each age, but in each case curves were also plotted from the velocity data. On this sample of children both methods produced almost identical curves.

TABLE 2

MEAN 'ADJUSTED' INCREMENTS OF RECUMBENT-LENGTH OVER 13-WEEK PERIODS OF CHILD STUDY CENTRE CHILDREN FROM 4 WEEKS TO 3 YEARS

(In inches to nearest $\mathbf{0} \cdot 1 \mathrm{inch}$, and $\mathrm{cm}$. to nearest $\mathrm{mm}$. 1 S.D. of mean given)

\begin{tabular}{|c|c|c|c|c|c|c|c|c|c|c|c|}
\hline \multirow{3}{*}{ Ages } & & \multicolumn{5}{|c|}{ Boys } & \multicolumn{5}{|c|}{ Girls } \\
\hline & & \multirow{2}{*}{ No. } & \multicolumn{2}{|c|}{ Inches } & \multicolumn{2}{|c|}{$\mathrm{Cm}$. } & \multirow{2}{*}{ No. } & \multicolumn{2}{|c|}{ Inches } & \multicolumn{2}{|c|}{$\mathrm{Cm}$. } \\
\hline & & & Mean & S.D. & Mean & S.D. & & Mean & S.D. & Mean & S.D. \\
\hline $\begin{array}{l}\text { 4-13 wks. } \ldots \\
13-26 \text { wks. . } \\
26-39 \text { wks. . } \\
39 \text { wks. }-1 \text { yr. } \\
1 \text { yr. }-18 \text { mos. } \\
18 \text { mos. }-2 \text { yrs. } \\
2-3 \text { yrs. } \quad . .\end{array}$ & $\begin{array}{l}\cdots \\
\cdots \\
\cdots \\
\cdots \\
\cdots \\
\cdots\end{array}$ & $\begin{array}{l}77 \\
87 \\
80 \\
71 \\
63 \\
51 \\
23\end{array}$ & $\begin{array}{l}3 \cdot 6 \\
2 \cdot 5 \\
1.9 \\
1.5 \\
1 \cdot 2 \\
1 \cdot 0 \\
0.8\end{array}$ & $\begin{array}{l}0.8 \\
0.5 \\
0.6 \\
0.5 \\
0.3 \\
0.3 \\
0.1\end{array}$ & $\begin{array}{l}9 \cdot 1 \\
6 \cdot 4 \\
4 \cdot 8 \\
3 \cdot 7 \\
3 \cdot 1 \\
2 \cdot 6 \\
2 \cdot 1\end{array}$ & $\begin{array}{l}2 \cdot 1 \\
1 \cdot 3 \\
1 \cdot 4 \\
1 \cdot 3 \\
0 \cdot 8 \\
0 \cdot 7 \\
0 \cdot 3\end{array}$ & $\begin{array}{l}66 \\
74 \\
69 \\
69 \\
58 \\
45 \\
20\end{array}$ & $\begin{array}{l}3 \cdot 4 \\
2 \cdot 6 \\
1 \cdot 9 \\
1 \cdot 5 \\
1 \cdot 3 \\
1 \cdot 0 \\
0 \cdot 8\end{array}$ & $\begin{array}{l}0.6 \\
0 \cdot 6 \\
0 \cdot 5 \\
0 \cdot 6 \\
0 \cdot 4 \\
0 \cdot 2 \\
0 \cdot 1\end{array}$ & $\begin{array}{l}8 \cdot 6 \\
6 \cdot 7 \\
4 \cdot 7 \\
3 \cdot 8 \\
3 \cdot 2 \\
2 \cdot 6 \\
2 \cdot 2\end{array}$ & $\begin{array}{l}1 \cdot 6 \\
1 \cdot 5 \\
1 \cdot 3 \\
1 \cdot 5 \\
0 \cdot 9 \\
0 \cdot 6 \\
0 \cdot 3\end{array}$ \\
\hline
\end{tabular}




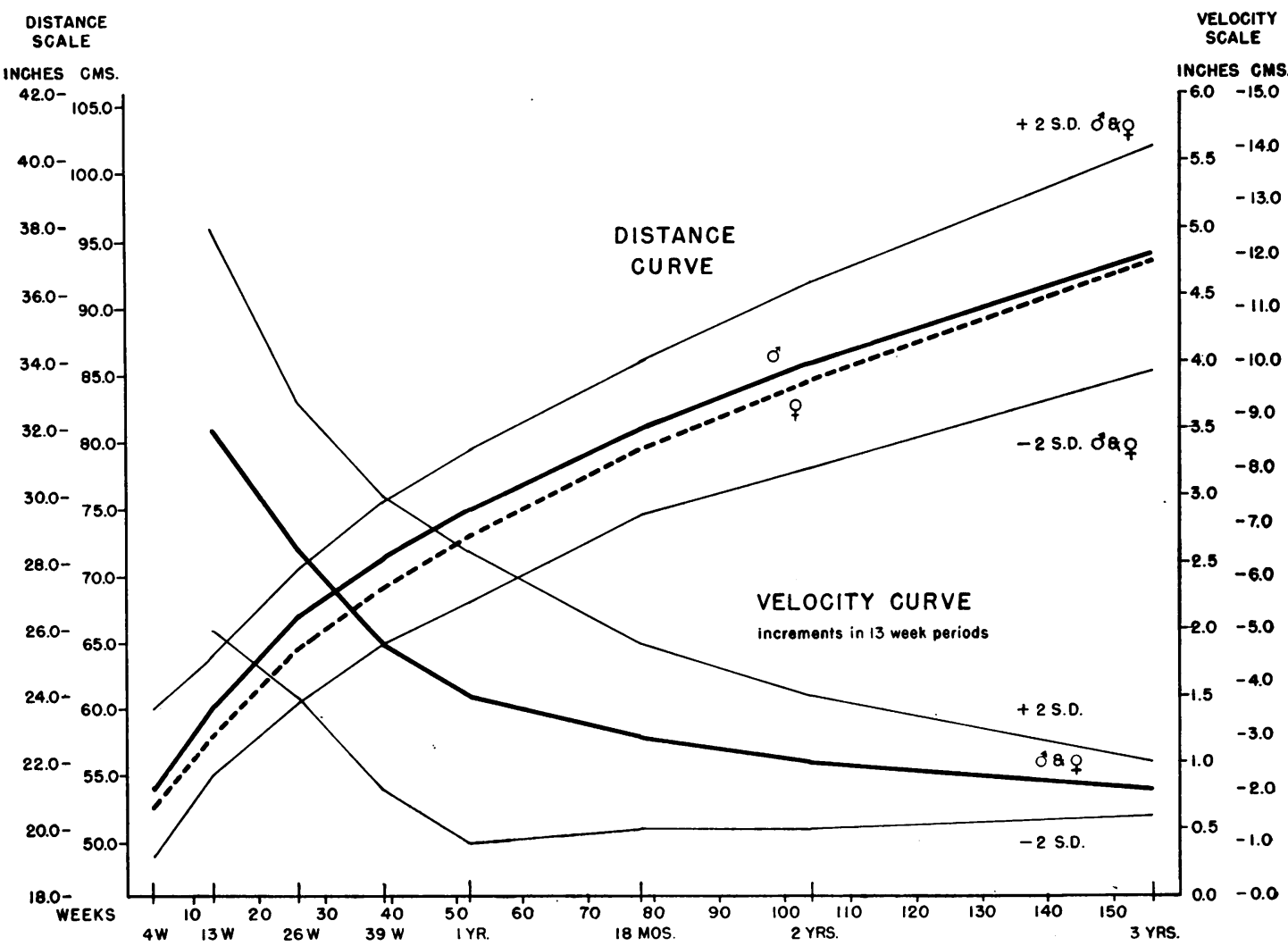

Fig. 1.-Recumbent-length: distance and velocity curves. In the distance curves, for simplicity, boys and girls are combined for \pm 2 S.D. curves. Boys and girls are combined to give the velocity curves, since their increments are so very similar.

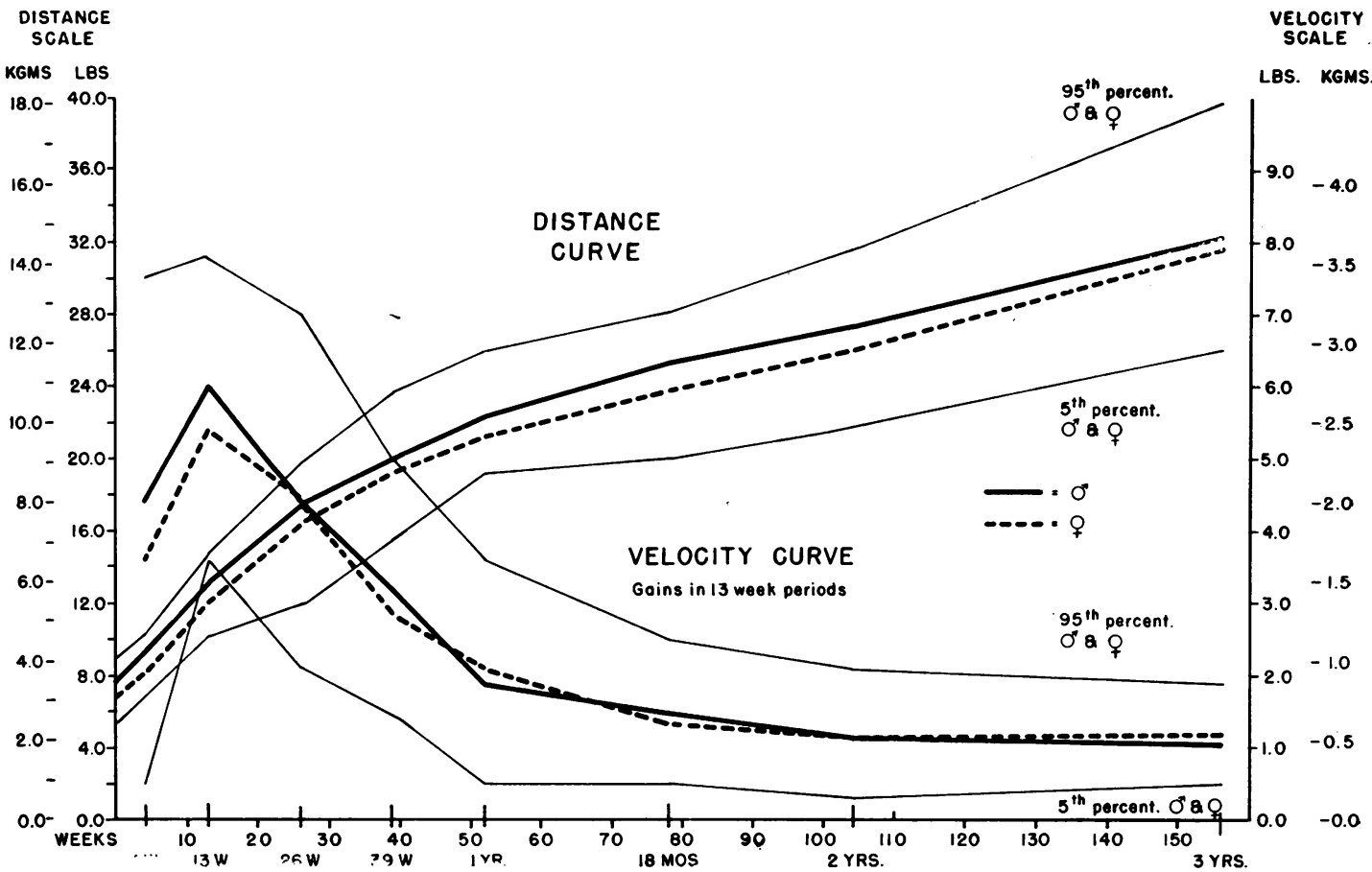

FIG. 2.-Weight: distance and velocity curves. For simplicity, boys and girls are combined to give the 95 th and 5 th percentiles. 
TABLE 3

MEAN WEIGHT OF CHILD STUDY CENTRE CHILDREN AT BIRTH TO 3 YEARS

(In lb. to nearest $0.1 \mathrm{lb}$., and $\mathrm{kg}$. to nearest $10 \mathrm{~g}$.)

\begin{tabular}{|c|c|c|c|c|c|c|c|c|c|c|c|c|}
\hline \multicolumn{13}{|c|}{ Boys } \\
\hline \multirow{3}{*}{\multicolumn{3}{|c|}{ Ages }} & \multirow{3}{*}{$\begin{array}{l}\text { No. of } \\
\text { Subjects }\end{array}$} & \multicolumn{7}{|c|}{ Percentiles } & \multirow{2}{*}{\multicolumn{2}{|c|}{ Mean Weight }} \\
\hline & & & & 1 & 5 & 20 & 50 & 80 & 95 & 99 & & \\
\hline & & & & \multicolumn{7}{|c|}{ Weight (lb.) } & Lb. & Kg. \\
\hline $\begin{array}{l}\text { At birth } \\
4 \text { wks. } \\
13 \text { wks. } \\
26 \text { wks. } \\
39 \text { wks. } \\
1 \text { yr. } \\
18 \text { mos. } \\
2 \text { yrs. } \\
3 \text { yrs. }\end{array}$ & $\begin{array}{l}. \\
\therefore \\
\therefore \\
\therefore \\
\therefore \\
\therefore\end{array}$ & $\begin{array}{c}. . \\
. \\
. \\
. \\
. \\
. \\
. \\
.\end{array}$ & $\begin{array}{l}96 \\
82 \\
96 \\
94 \\
84 \\
81 \\
74 \\
57 \\
33\end{array}$ & $\begin{array}{r}5 \cdot 6 \\
6 \cdot 4 \\
7 \cdot 8 \\
10 \cdot 9 \\
13 \cdot 0 \\
14 \cdot 6 \\
18 \cdot 9 \\
21 \cdot 7 \\
25 \cdot 0\end{array}$ & $\begin{array}{r}5 \cdot 9 \\
7 \cdot 0 \\
10 \cdot 8 \\
14 \cdot 6 \\
16 \cdot 3 \\
17 \cdot 0 \\
20 \cdot 1 \\
22 \cdot 0 \\
25 \cdot 1\end{array}$ & $\begin{array}{r}6 \cdot 7 \\
7 \cdot 9 \\
11 \cdot 6 \\
16 \cdot 0 \\
18 \cdot 6 \\
20 \cdot 3 \\
22 \cdot 8 \\
25 \cdot 3 \\
27 \cdot 7\end{array}$ & $\begin{array}{r}7 \cdot 7 \\
9 \cdot 1 \\
12 \cdot 9 \\
17 \cdot 6 \\
20 \cdot 9 \\
23 \cdot 0 \\
25 \cdot 9 \\
27 \cdot 6 \\
32 \cdot 6\end{array}$ & $\begin{array}{r}8 \cdot 4 \\
9 \cdot 9 \\
14 \cdot 6 \\
19 \cdot 0 \\
22 \cdot 4 \\
24 \cdot 5 \\
27.7 \\
30 \cdot 1 \\
35 \cdot 4\end{array}$ & $\begin{array}{r}9 \cdot 5 \\
10 \cdot 8 \\
15 \cdot 7 \\
19 \cdot 9 \\
24 \cdot 1 \\
26 \cdot 3 \\
28 \cdot 5 \\
31 \cdot 9 \\
39 \cdot 5\end{array}$ & $\begin{array}{l}11 \cdot 2 \\
12 \cdot 2 \\
16 \cdot 9 \\
21 \cdot 9 \\
26 \cdot 6 \\
27 \cdot 0 \\
30 \cdot 4 \\
32 \cdot 6 \\
50 \cdot 2\end{array}$ & $\begin{array}{r}7 \cdot 6 \\
8 \cdot 9 \\
13 \cdot 1 \\
17 \cdot 4 \\
20 \cdot 4 \\
22 \cdot 4 \\
25 \cdot 4 \\
27 \cdot 5 \\
32 \cdot 2\end{array}$ & $\begin{array}{r}3 \cdot 46 \\
4 \cdot 05 \\
5 \cdot 91 \\
7 \cdot 91 \\
9 \cdot 25 \\
10 \cdot 15 \\
11 \cdot 50 \\
12 \cdot 48 \\
14 \cdot 60\end{array}$ \\
\hline
\end{tabular}

Girls

\begin{tabular}{|c|c|c|c|c|c|c|c|c|c|c|c|c|}
\hline & & & & \multicolumn{7}{|c|}{ Percentiles } & \multirow{2}{*}{ Mean } & \multirow{2}{*}{ Neight } \\
\hline \multirow{2}{*}{\multicolumn{3}{|c|}{ Ages }} & No. of & 1 & 5 & 20 & 50 & 80 & 95 & 99 & & \\
\hline & & & & \multicolumn{7}{|c|}{ Weight (lb.) } & Lb. & Kg. \\
\hline $\begin{array}{l}\text { At birth } \\
4 \text { wks. } \\
13 \text { wks. } \\
26 \text { wks. } \\
39 \text { wks. } \\
1 \text { yr. } \\
18 \text { mos. } \\
2 \text { yrs. } \\
3 \text { yrs. }\end{array}$ & $\begin{array}{l}. \\
\ldots \\
\ldots \\
\ldots \\
\ldots \\
\ldots\end{array}$ & $\begin{array}{l}. . \\
\cdots \\
\cdots \\
\cdots \\
\cdots \\
\cdots \\
\cdots\end{array}$ & $\begin{array}{l}93 \\
77 \\
84 \\
81 \\
78 \\
78 \\
67 \\
57 \\
22\end{array}$ & $\begin{array}{r}4 \cdot 6 \\
6 \cdot 2 \\
8 \cdot 4 \\
12 \cdot 6 \\
14 \cdot 0 \\
15 \cdot 8 \\
18 \cdot 6 \\
20 \cdot 8 \\
24 \cdot 1\end{array}$ & $\begin{array}{r}5 \cdot 1 \\
6 \cdot 5 \\
9 \cdot 5 \\
13 \cdot 1 \\
14 \cdot 8 \\
17 \cdot 4 \\
19 \cdot 8 \\
21 \cdot 5 \\
27 \cdot 2\end{array}$ & $\begin{array}{r}6 \cdot 2 \\
7 \cdot 4 \\
10 \cdot 9 \\
14 \cdot 7 \\
17 \cdot 1 \\
19 \cdot 2 \\
21 \cdot 9 \\
23 \cdot 6 \\
28 \cdot 8\end{array}$ & $\begin{array}{r}7 \cdot 2 \\
8 \cdot 2 \\
12 \cdot 2 \\
16 \cdot 5 \\
19 \cdot 3 \\
21 \cdot 0 \\
23 \cdot 9 \\
25 \cdot 8 \\
30 \cdot 9\end{array}$ & $\begin{array}{r}8 \cdot 0 \\
9 \cdot 1 \\
13 \cdot 0 \\
17 \cdot 9 \\
21 \cdot 2 \\
23 \cdot 1 \\
26 \cdot 2 \\
28 \cdot 3 \\
34 \cdot 3\end{array}$ & $\begin{array}{r}8 \cdot 8 \\
9 \cdot 7 \\
13 \cdot 7 \\
19 \cdot 7 \\
23 \cdot 6 \\
25 \cdot 9 \\
28 \cdot 0 \\
31 \cdot 3 \\
40 \cdot 3\end{array}$ & $\begin{array}{r}9 \cdot 4 \\
10 \cdot 7 \\
14 \cdot 7 \\
21 \cdot 0 \\
25 \cdot 7 \\
26 \cdot 9 \\
31 \cdot 3 \\
32 \cdot 6 \\
43 \cdot 3\end{array}$ & $\begin{array}{r}7 \cdot 1 \\
8 \cdot 2 \\
11 \cdot 9 \\
16 \cdot 4 \\
19 \cdot 2 \\
21 \cdot 3 \\
23 \cdot 9 \\
26 \cdot 1 \\
31 \cdot 8\end{array}$ & $\begin{array}{r}3 \cdot 23 \\
3 \cdot 73 \\
5 \cdot 39 \\
7 \cdot 43 \\
8 \cdot 69 \\
9 \cdot 64 \\
10 \cdot 85 \\
11 \cdot 81 \\
14 \cdot 43\end{array}$ \\
\hline
\end{tabular}

TABLE 4

'ADJUSTED' MEAN INCREMENTS OF WEIGHT GAIN OVER 13-WEEK PERIODS OF CHILD STUDY CENTRE CHILDREN FROM BIRTH TO 3 YEARS

(In lb. to nearest $0.1 \mathrm{lb}$., and $\mathrm{kg}$. to nearest $10 \mathrm{~g}$.)

\begin{tabular}{|c|c|c|c|c|c|c|c|c|c|c|c|}
\hline \multicolumn{12}{|c|}{ Boys } \\
\hline \multirow{3}{*}{ Ages } & & \multirow{3}{*}{$\begin{array}{l}\text { No. of } \\
\text { Subjects }\end{array}$} & \multicolumn{7}{|c|}{ Percentiles } & \multirow{2}{*}{\multicolumn{2}{|c|}{ Mean Increments }} \\
\hline & & & 1 & 5 & 20 & 50 & 80 & 95 & 99 & & \\
\hline & & & \multicolumn{7}{|c|}{ Increments (lb.) } & Lb. & Kg. \\
\hline $\begin{array}{l}\text { Birth }-4 \text { wks. } \\
4-13 \text { wks. } \\
13-26 \text { wks. } . . \\
26-39 \text { wks. } \\
39-52 \text { wks. ... } \\
11 \text { yr.-18 mos. } \\
18 \text { mos. } 2 \text { yrs. } \\
2-3 \text { yrs. }\end{array}$ & $\begin{array}{l}. . \\
\cdots \\
\cdots \\
. . \\
. . \\
. .\end{array}$ & $\begin{array}{l}73 \\
77 \\
87 \\
80 \\
72 \\
60 \\
51 \\
29\end{array}$ & $\begin{array}{c}-2 \cdot 2 \\
3 \cdot 0 \\
1 \cdot 3 \\
0 \cdot 7 \\
-1 \cdot 3 \\
-0 \cdot 05 \\
0 \cdot 2 \\
0 \cdot 3\end{array}$ & $\begin{array}{l}0 \cdot 3 \\
4 \cdot 1 \\
2 \cdot 1 \\
1 \cdot 5 \\
0 \cdot 2 \\
0 \cdot 6 \\
0 \cdot 5 \\
0 \cdot 5\end{array}$ & $\begin{array}{l}2 \cdot 8 \\
4.8 \\
3.4 \\
2.4 \\
1.2 \\
0.9 \\
0.7 \\
0.8\end{array}$ & $\begin{array}{l}4 \cdot 5 \\
5 \cdot 7 \\
4 \cdot 4 \\
3 \cdot 1 \\
2 \cdot 0 \\
1 \cdot 4 \\
1 \cdot 2 \\
1 \cdot 1\end{array}$ & $\begin{array}{l}5 \cdot 9 \\
7 \cdot 4 \\
5 \cdot 4 \\
4 \cdot 1 \\
2 \cdot 4 \\
2 \cdot 0 \\
1 \cdot 5 \\
1 \cdot 3\end{array}$ & $\begin{array}{l}8 \cdot 2 \\
8 \cdot 4 \\
6 \cdot 8 \\
4 \cdot 9 \\
3 \cdot 8 \\
2 \cdot 4 \\
2 \cdot 1 \\
1 \cdot 7\end{array}$ & $\begin{array}{r}9 \cdot 7 \\
12 \cdot 2 \\
7 \cdot 5 \\
7 \cdot 2 \\
4 \cdot 3 \\
2 \cdot 6 \\
2 \cdot 4 \\
2 \cdot 4\end{array}$ & $\begin{array}{l}4 \cdot 4 \\
6 \cdot 1 \\
4 \cdot 4 \\
3 \cdot 2 \\
1 \cdot 9 \\
1 \cdot 5 \\
1 \cdot 2 \\
1 \cdot 1\end{array}$ & $\begin{array}{l}2 \cdot 00 \\
2 \cdot 74 \\
2 \cdot 01 \\
1 \cdot 45 \\
0 \cdot 86 \\
0.66 \\
0.53 \\
0.49\end{array}$ \\
\hline \multicolumn{12}{|c|}{ Girls } \\
\hline \multirow{3}{*}{ Ages } & & \multirow{3}{*}{$\begin{array}{l}\text { No. of } \\
\text { Subjects }\end{array}$} & \multicolumn{7}{|c|}{ Percentiles } & \multirow{2}{*}{\multicolumn{2}{|c|}{ Mean Increments }} \\
\hline & & & 1 & 5 & 20 & 50 & 80 & 95 & 99 & & \\
\hline & & & \multicolumn{7}{|c|}{ Increments (lb.) } & Lb. & Kg. \\
\hline $\begin{array}{l}\text { Birth }-4 \text { wks. } \\
4-13 \text { wks. } \\
13-26 \text { wks. } . . \\
26-39 \text { wks. } \\
38-52 \text { wks. } . . \\
1 \text { yr. } 18 \text { mos. } \\
18 \text { mos. }-2 \text { yrs. } \\
2-3 \text { yrs. }\end{array}$ & $\begin{array}{l}. \\
. \\
\cdots \\
\cdots \\
\cdots \\
\cdots \\
.\end{array}$ & $\begin{array}{l}72 \\
65 \\
73 \\
69 \\
69 \\
59 \\
48 \\
21\end{array}$ & $\begin{array}{r}-1 \cdot 2 \\
2.5 \\
1.1 \\
0.2 \\
0.1 \\
-0.2 \\
-0.4 \\
0.5\end{array}$ & $\begin{array}{l}0 \cdot 7 \\
3 \cdot 2 \\
2 \cdot 1 \\
1 \cdot 3 \\
0 \cdot 9 \\
0 \cdot 3 \\
0 \cdot 2 \\
0 \cdot 6\end{array}$ & $\begin{array}{l}2 \cdot 1 \\
4 \cdot 3 \\
3 \cdot 3 \\
2 \cdot 0 \\
1 \cdot 4 \\
0 \cdot 9 \\
0 \cdot 7 \\
0 \cdot 7\end{array}$ & $\begin{array}{l}3 \cdot 5 \\
5 \cdot 1 \\
4 \cdot 4 \\
2 \cdot 6 \\
2 \cdot 0 \\
1 \cdot 3 \\
1 \cdot 2 \\
1 \cdot 0\end{array}$ & $\begin{array}{l}5 \cdot 0 \\
6 \cdot 7 \\
5 \cdot 8 \\
3 \cdot 9 \\
2 \cdot 7 \\
1 \cdot 9 \\
1 \cdot 7 \\
1 \cdot 4\end{array}$ & $\begin{array}{l}6 \cdot 8 \\
7 \cdot 2 \\
7 \cdot 2 \\
5 \cdot 4 \\
3 \cdot 4 \\
4 \cdot 6 \\
2 \cdot 1 \\
2 \cdot 2\end{array}$ & $\begin{array}{l}7 \cdot 8 \\
8 \cdot 4 \\
7 \cdot 6 \\
6 \cdot 0 \\
4 \cdot 4 \\
3 \cdot 0 \\
2 \cdot 4 \\
2 \cdot 7\end{array}$ & $\begin{array}{l}3 \cdot 6 \\
5 \cdot 3 \\
4 \cdot 5 \\
2 \cdot 9 \\
2 \cdot 1 \\
1 \cdot 4 \\
1 \cdot 2 \\
1 \cdot 2\end{array}$ & $\begin{array}{l}1.64 \\
2.42 \\
2.05 \\
1.30 \\
0.93 \\
0.62 \\
0.53 \\
0.52\end{array}$ \\
\hline
\end{tabular}


Discussion. While it is well known that boys are taller and heavier than girls, it is interesting to see how closely similar they are in increments (rate of growth) of recumbent-length and weight in this age period, except in the case of weight gain between birth and 3 months. Their birth weight and early recumbent-length differences are maintained.

From other studies (example: Simmons, 1944) it appeared that the size of the newborn baby is not related strongly to his size at, say, 2 years, and Tanner, Healy, Lockhart, MacKenzie and Whitehouse (1956) have shown that birth size is only very slightly related to adult size. It would seem that the genetically determined factor in the growth pattern of the child does not start to become evident until after 1 year of age and is quite apparent at 2 years, presumably the prenatal environmental influences having now been mainly eliminated. Tanner found a high relationship between adult size and size at 3 years.

The London data were examined to verify this hypothesis. Since weight is subject so much to the vicissitudes of life in this period, recumbent-length only was examined.

To check the possible effect of illness on these assumptions the groups were subdivided into children who had been ( $a$ ) ill and not hospitalized, (b) hospitalized and (c) not ill at all. The correlations remained almost identically the same. Table 5
TABLE 5

CORRELATIONS BETWEEN RECUMBENT-LENGTHS OF CHILD STUDY CENTRE CHILDREN AT 4 WEEKS AND 1 YEAR AND AT 1 YEAR AND 2 YEARS

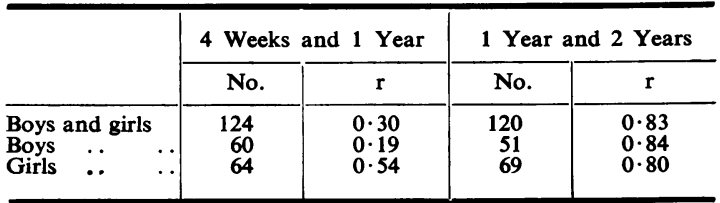

shows there is little relationship between recumbentlength at 4 weeks and 1 year, but a close relationship between 1 year and 2 years.

Regarding rate of growth, increments of recumbent-length between 4-13 weeks and 1 year18 months were examined for relationships. Table 6 shows there is a very low relationship and confirms Tanner's (1956) observation that rate and size seem practically independent.

The Oxford, Aberdeen and another London set of

TABLE 6

CORRELATIONS OF RECUMBENT-LENGTH INCREMENTS OF CHILD STUDY CENTRE CHILDREN BETWEEN 4-13 WEEKS AND 1 YEAR-18 MONTHS

\begin{tabular}{|c|c|c|c|c|}
\hline & & & No. & $\mathbf{r}$ \\
\hline 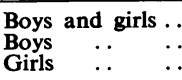 & $\begin{array}{l}\ldots \\
\ldots \\
\ldots\end{array}$ & $\begin{array}{c}\cdots \\
\cdots \\
\cdots\end{array}$ & $\begin{array}{r}124 \\
54 \\
50\end{array}$ & $\begin{array}{l}0 \cdot 08 \\
0 \cdot 07 \\
0 \cdot 23\end{array}$ \\
\hline
\end{tabular}

TABLE 7

MEAN COMPARATIVE LENGTHS

(In inches to nearest 0.1 inch)

\begin{tabular}{|c|c|c|c|c|c|c|c|c|}
\hline \multirow[t]{2}{*}{ Ages } & \multicolumn{2}{|c|}{$\begin{array}{l}\text { London, } 1926 \\
\text { (Myers, 1926) }\end{array}$} & \multicolumn{2}{|c|}{$\begin{array}{c}\text { Aberdeen } \\
\text { (Low, 1952) }\end{array}$} & \multicolumn{2}{|c|}{$\begin{array}{c}\text { Oxford } \\
\text { (Acheson } \text { et al., 1955) }\end{array}$} & \multicolumn{2}{|c|}{$\begin{array}{c}\text { London, } 1952 \\
\text { (Child Study Centre) }\end{array}$} \\
\hline & Boys & Girls & Boys & Girls & Boys & Girls & Boys & Girls \\
\hline $\begin{array}{l}\text { Birth } \\
4 \text { wks. } \\
13 \text { wks. } \\
26 \text { wks. } \\
39 \text { wks. } \\
1 \text { yr. } \\
18 \text { mos. } \\
2 \text { yrs. } \\
2 \frac{1}{2} \text { yrs. } \\
3 \text { yrs. }\end{array}$ & $\begin{array}{l}-\overline{21 \cdot 0} \\
23 \cdot 0 \\
25 \cdot 0 \\
26 \cdot 2 \\
27 \cdot 9 \\
31 \cdot 0 \\
32 \cdot 4 \\
34 \cdot 0 \\
36 \cdot 1\end{array}$ & $\begin{array}{l}-\overline{20 \cdot 9} \\
22 \cdot 7 \\
25 \cdot 3 \\
26 \cdot 8 \\
27 \cdot 5 \\
30 \cdot 6 \\
32 \cdot 1 \\
33 \cdot 4 \\
35 \cdot 4\end{array}$ & $\begin{array}{l}19 \cdot 6 \\
\overline{-} \\
\overline{-} \\
28 \cdot 9 \\
\overline{32 \cdot 9} \\
\overline{36 \cdot 2}\end{array}$ & $\begin{array}{l}19 \cdot 5 \\
\overline{-} \\
\overline{-} \\
2 \overline{28 \cdot 6} \\
\overline{32 \cdot 9} \\
\overline{36 \cdot 4}\end{array}$ & $\begin{array}{l}\bar{Z} \\
\bar{Z} \\
\bar{Z} \\
31 \cdot 9^{*} \\
34 \cdot 0^{*} \\
35 \cdot 6^{*} \\
37 \cdot 3^{*}\end{array}$ & $\begin{array}{l}\bar{Z} \\
\bar{Z} \\
\overline{-} \\
31 \cdot 1^{*} \\
33 \cdot 2^{*} \\
35 \cdot 1^{*} \\
36 \cdot 7^{*}\end{array}$ & $\begin{array}{l}\overline{21 \cdot 2} \\
23 \cdot 7 \\
26 \cdot 3 \\
28 \cdot 1 \\
29 \cdot 5 \\
32 \cdot 0 \\
34 \cdot 0 \\
37 \cdot 1\end{array}$ & $\begin{array}{l}2 \overline{20 \cdot 7} \\
22 \cdot 9 \\
25 \cdot 5 \\
27 \cdot 3 \\
28 \cdot 9 \\
31 \cdot 4 \\
33 \cdot 3 \\
3 \overline{37 \cdot 0}\end{array}$ \\
\hline
\end{tabular}

* Standing height.

TABLE 8

MEAN COMPARATIVE INCREMENTS IN LENGTH

(In inches to nearest 0.1 inch)

\begin{tabular}{|c|c|c|c|c|c|c|}
\hline \multirow{2}{*}{ Ages } & \multicolumn{3}{|c|}{ Boys } & \multicolumn{3}{|c|}{ Girls } \\
\hline & Aberdeen & Oxford & London, 1952 & Aberdeen & Oxford & London, 1952 \\
\hline $\begin{array}{l}1 \text { yr. }-2 \text { yrs. } \\
18 \text { mos. }-2 \text { yrs. } \\
2-3 \text { yrs. }\end{array}$ & $\frac{1 \cdot 0}{0 \cdot 8}$ & $\begin{array}{l}\overline{1 \cdot 0^{*}} \\
0 \cdot 8^{*}\end{array}$ & $\begin{array}{l}1 \cdot 1 \\
1 \cdot 0 \\
0 \cdot 8\end{array}$ & $\frac{1 \cdot 1}{0 \cdot 9}$ & $\begin{array}{l}\overline{1 \cdot 0^{*}} \\
0 \cdot 9^{*}\end{array}$ & $\begin{array}{l}1 \cdot 1 \\
1 \cdot 0 \\
0 \cdot 9\end{array}$ \\
\hline
\end{tabular}

* Standing height. 
TABLE 9

MEAN COMPARATIVE WEIGHTS

(In lb. to nearest $0.1 \mathrm{lb}$.)

\begin{tabular}{|c|c|c|c|c|c|c|c|c|c|c|c|}
\hline \multirow[t]{2}{*}{ Ages } & & \multicolumn{2}{|c|}{$\begin{array}{l}\text { London, } 1926 \\
\text { (Myers, 1926) }\end{array}$} & \multicolumn{2}{|c|}{$\begin{array}{c}\text { Aberdeen } \\
\text { (Low, 1952) }\end{array}$} & \multicolumn{2}{|c|}{$\begin{array}{l}\text { London, } 1947 \\
\text { (Gore and Palmer, 1949) }\end{array}$} & \multicolumn{2}{|c|}{$\begin{array}{c}\text { Oxford } \\
\text { (Acheson } \text { et al., 1955) }\end{array}$} & \multicolumn{2}{|c|}{$\begin{array}{c}\text { London, } 1952 \\
\text { (Child Study Centre) }\end{array}$} \\
\hline & & Boys & Girls & Boys & Girls & Boys & Girls & Boys & Girls & Boys & Girls \\
\hline $\begin{array}{l}\text { Birth } \\
4 \text { wks. } \\
13 \text { wks. } \\
26 \text { wks. } \\
39 \text { wks. } \\
1 \text { yr. } \\
18 \text { mos. } \\
2 \text { yrs. } \\
2 \frac{1}{2} \text { yrs. } \\
3 \text { yrs. }\end{array}$ & $\begin{array}{c}\ldots \\
\ldots \\
\ldots \\
\cdots \\
\ldots \\
\cdots \\
\cdots \\
\cdots \\
\cdots\end{array}$ & $\begin{array}{l}\overline{8 \cdot 3} \\
11 \cdot 5 \\
15 \cdot 4 \\
18 \cdot 1 \\
20 \cdot 5 \\
22 \cdot 7 \\
26 \cdot 5 \\
28 \cdot 2 \\
30 \cdot 2\end{array}$ & $\begin{array}{r}\overline{8 \cdot 2} \\
10 \cdot 7 \\
15 \cdot 8 \\
18 \cdot 1 \\
19 \cdot 5 \\
22 \cdot 1 \\
25 \cdot 0 \\
24 \cdot 7 \\
29 \cdot 3\end{array}$ & $\begin{array}{l}7 \cdot 0 \\
\overline{-} \\
\overline{-} \\
20 \cdot 6 \\
\overline{25 \cdot 2} \\
\overline{28 \cdot 7}\end{array}$ & $\begin{array}{l}6 \cdot 9 \\
\bar{Z} \\
\bar{E} \\
\overline{19 \cdot 9} \\
\overline{24 \cdot 6} \\
\overline{29 \cdot 4}\end{array}$ & $\begin{array}{r}\overline{8 \cdot 6} \\
13 \cdot 1 \\
17 \cdot 5 \\
20 \cdot 5 \\
22 \cdot 6 \\
25 \cdot 5 \\
28 \cdot 3 \\
30 \cdot 8 \\
32 \cdot 8\end{array}$ & $\begin{array}{r}\overline{8} \cdot 5 \\
12 \cdot 2 \\
16 \cdot 4 \\
19 \cdot 3 \\
21 \cdot 0 \\
24 \cdot 1 \\
26 \cdot 9 \\
29 \cdot 5 \\
31 \cdot 6\end{array}$ & $\begin{array}{l}7 \cdot 6 \\
\overline{17 \cdot 5} \\
\overline{17 \cdot 5} \\
25 \cdot 7 \\
27 \cdot 8 \\
29 \cdot 9 \\
32 \cdot 2\end{array}$ & $\begin{array}{l}7 \cdot 3 \\
\overline{1} \overline{16 \cdot 1} \\
\overline{21 \cdot 1} \\
23 \cdot 9 \\
26 \cdot 1 \\
28 \cdot 6 \\
31 \cdot 0\end{array}$ & $\begin{array}{r}7 \cdot 6 \\
8 \cdot 9 \\
13 \cdot 1 \\
17 \cdot 4 \\
20 \cdot 4 \\
22 \cdot 4 \\
25 \cdot 4 \\
27 \cdot 5 \\
32 \cdot 2\end{array}$ & $\begin{array}{r}7 \cdot 1 \\
8 \cdot 2 \\
11 \cdot 9 \\
16 \cdot 4 \\
19 \cdot 2 \\
21 \cdot 3 \\
23 \cdot 9 \\
26 \cdot 1 \\
31 \cdot 8\end{array}$ \\
\hline
\end{tabular}

TABLE 10

COMPARATIVE MEAN INCREMENTS OF WEIGHT

(In lb. to nearest $0.1 \mathrm{lb}$.)

\begin{tabular}{|c|c|c|c|c|c|c|c|c|c|}
\hline \multirow[t]{2}{*}{ Ages } & & \multicolumn{2}{|c|}{$\begin{array}{c}\text { Aberdeen } \\
\text { (Low, 1952) }\end{array}$} & \multicolumn{2}{|c|}{$\begin{array}{l}\text { London, } 1947 \\
\text { (Gore and Palmer, 1949) }\end{array}$} & \multicolumn{2}{|c|}{$\begin{array}{l}\text { Oxford } \\
\text { (Acheson et al., 1955) }\end{array}$} & \multicolumn{2}{|c|}{$\begin{array}{c}\text { London, } 1952 \\
\text { (Child Study Centre) }\end{array}$} \\
\hline & & Boys & Girls & Boys & Girls & Boys & Girls & Boys & Girls \\
\hline $\begin{array}{l}\text { Birth-4 wks. } \\
4-13 \text { wks. } \\
13-26 \text { wks. } \\
26-39 \text { wks. } \\
39 \text { wks. }-1 \text { yr. } \\
1 \text { yr. }-18 \text { mos. } \\
1 \text { yr. }-2 \text { yrs. } \\
18 \text { mos. }-2 \text { yrs. } \\
2-3 \text { yrs. }\end{array}$ & $\begin{array}{l}\cdots \\
\cdots \\
\cdots \\
\cdots \\
\cdots \\
\cdots \\
\cdots \\
\cdots\end{array}$ & $\begin{array}{l}\bar{z} \\
\bar{z} \\
\overline{1.2} \\
\overline{0.9}\end{array}$ & $\begin{array}{l}E \\
\bar{E} \\
\bar{E} \\
\overline{1 \cdot 2} \\
1 \cdot 2\end{array}$ & $\begin{array}{l}\overline{6} \cdot 5 \\
4 \cdot 4 \\
3 \cdot 0 \\
2 \cdot 1 \\
1 \cdot 5 \\
1 \cdot 4 \\
1 \cdot 2 \\
1 \cdot 1\end{array}$ & $\begin{array}{l}\overline{5} \cdot 3 \\
4 \cdot 2 \\
2 \cdot 9 \\
1 \cdot 7 \\
1 \cdot 5 \\
1 \cdot 5 \\
1 \cdot 4 \\
1 \cdot 2\end{array}$ & $\begin{array}{l}- \\
- \\
- \\
1 \cdot 4 \\
1 \cdot 3 \\
1 \cdot 2 \\
1 \cdot 1\end{array}$ & $\begin{array}{l}- \\
- \\
\bar{Z} \\
1 \cdot 4 \\
1 \cdot 2 \\
1 \cdot 1 \\
1 \cdot 1\end{array}$ & $\begin{array}{l}4 \cdot 4 \\
6 \cdot 1 \\
4 \cdot 4 \\
3 \cdot 2 \\
1 \cdot 9 \\
1 \cdot 5 \\
1 \cdot 3 \\
1 \cdot 2 \\
1 \cdot 1\end{array}$ & $\begin{array}{l}3 \cdot 6 \\
5 \cdot 3 \\
4 \cdot 5 \\
2 \cdot 9 \\
2 \cdot 1 \\
1 \cdot 4 \\
1 \cdot 2 \\
1 \cdot 2 \\
1 \cdot 2\end{array}$ \\
\hline
\end{tabular}

children are compared in means in Tables 7 and 8. The Aberdeen and London data in Table 8 are true mean increments. The Oxford increments have been obtained by subtracting mean heights at the ages stated; they are thus an approximation only.

Comparative weights are given in Tables 9 and 10. Because of the arrangement of age groups in Table 10 the London 1926 data did not permit increments to be obtained. The London 1947 and Oxford 1948 increments in this table have been obtained by subtracting the means at the ages stated and are thus approximations; the Aberdeen and London 1952 increments are true increments.

Tables 7, 8, 9 and 10 show that, although the samples are not strictly comparable, the trend of children to become taller and heavier as time goes on is apparently maintained (Annotation in Lancet, 1955; Tanner, 1955). This is apparently due to the consistent biological fact that children are reaching physical maturity earlier and earlier. Skeletal maturity assessments upon the early samples of children would be most interesting had they been

TABLE 11

MEAN CROWN-RUMP MEASUREMENTS OF CHILD STUDY CENTRE CHILDREN AGED 4 WEEKS TO 3 YEARS (In inches to nearest $0 \cdot 1 \mathrm{inch}$, and $\mathrm{cm}$. to nearest $\mathrm{mm}$. S.D. $=1$ standard deviation of mean)

\begin{tabular}{|c|c|c|c|c|c|c|c|c|c|c|c|c|}
\hline & & & \multicolumn{5}{|c|}{ Boys } & \multicolumn{5}{|c|}{ Girls } \\
\hline \multirow{2}{*}{\multicolumn{3}{|c|}{ Ages }} & \multirow{2}{*}{ No. } & \multicolumn{2}{|c|}{ Inches } & \multicolumn{2}{|c|}{$\mathrm{Cm}}$. & \multirow{2}{*}{ No. } & \multicolumn{2}{|c|}{ Inches } & \multicolumn{2}{|c|}{$\mathrm{Cm}$. } \\
\hline & & & & Mean & S.D. & Mean & S.D. & & Mean & S.D. & Mean & S.D. \\
\hline $\begin{array}{l}4 \text { wks. } \\
13 \text { wks. } \\
26 \text { wks. } \\
39 \text { wks. } \\
11 \text { yr. } \\
18 \text { mos. } \\
2 \text { yrs. } \\
3 \text { yrs. }\end{array}$ & $\begin{array}{l}\ldots \\
\cdots \\
\cdots \\
\cdots \\
\cdots \\
\cdots\end{array}$ & \begin{tabular}{l|}
. \\
$\cdots$ \\
$\cdots$ \\
$\cdots$ \\
$\cdots$ \\
. \\
..
\end{tabular} & $\begin{array}{l}82 \\
95 \\
94 \\
84 \\
79 \\
85 \\
55 \\
31\end{array}$ & $\begin{array}{l}13 \cdot 8 \\
15.4 \\
17.0 \\
18 \cdot 0 \\
18 \cdot 7 \\
19 \cdot 8 \\
20 \cdot 7 \\
22 \cdot 2\end{array}$ & $\begin{array}{l}0.7 \\
0.7 \\
0.6 \\
0.7 \\
0.6 \\
0.7 \\
0.9 \\
1.1\end{array}$ & $\begin{array}{l}35 \cdot 0 \\
39 \cdot 2 \\
43 \cdot 3 \\
45 \cdot 8 \\
47 \cdot 5 \\
50 \cdot 2 \\
52 \cdot 6 \\
56 \cdot 3\end{array}$ & $\begin{array}{l}1.7 \\
1.7 \\
1.6 \\
1.8 \\
1.6 \\
1.8 \\
2.4 \\
2.7\end{array}$ & $\begin{array}{l}77 \\
84 \\
81 \\
77 \\
77 \\
75 \\
53 \\
26\end{array}$ & $\begin{array}{l}13 \cdot 7 \\
14 \cdot 9 \\
16 \cdot 6 \\
17 \cdot 5 \\
18 \cdot 2 \\
19 \cdot 4 \\
20 \cdot 3 \\
21 \cdot 6\end{array}$ & $\begin{array}{l}0.5 \\
0.5 \\
0.5 \\
0.6 \\
0.8 \\
0.7 \\
0.7 \\
1 \cdot 1\end{array}$ & $\begin{array}{l}34 \cdot 3 \\
37 \cdot 9 \\
42 \cdot 1 \\
44 \cdot 5 \\
46 \cdot 2 \\
49 \cdot 3 \\
51 \cdot 6 \\
54 \cdot 9\end{array}$ & $\begin{array}{l}1 \cdot 3 \\
1 \cdot 2 \\
1 \cdot 3 \\
1 \cdot 5 \\
2 \cdot 0 \\
1.9 \\
1.7 \\
2 \cdot 7\end{array}$ \\
\hline
\end{tabular}


available. The Oxford 1948 and London 1952 data are very similar. It is also apparent how very similar are all the incremental figures irrespective of the date of the study. Again, rate of growth and size have been suggested to be independent.

\section{Other Measurements}

Upper Segment (or Crown-Rump Length) and Lower Segment (or L.ower-stem Length). The crown-rump measurement was obtained on the same measuring-board as for recumbent-length. The child's legs are raised vertically, the measurement

TABLE 12 MEASUREMENT IN 13-WEEK PERIODS OF CHILD STUDY

CENTRE CHILDREN AGED 4 WEEKS TO 3 YEARS (In inches to nearest $0 \cdot 1$ inch, and $\mathrm{cm}$. to nearest $\mathrm{mm}$.)

\begin{tabular}{|c|c|c|c|c|c|c|}
\hline \multirow{3}{*}{ Ages } & \multicolumn{3}{|c|}{ Boys } & \multicolumn{3}{|c|}{ Girls } \\
\hline & \multirow{2}{*}{ No. } & \multicolumn{2}{|c|}{$\begin{array}{l}\text { 'Adjusted' Mean } \\
\text { Increment }\end{array}$} & \multirow{2}{*}{ No. } & \multicolumn{2}{|c|}{$\begin{array}{l}\text { 'Adjusted' Mean } \\
\text { Increment }\end{array}$} \\
\hline & & Inches & $\mathrm{Cm}$. & & Inches & $\mathrm{Cm}$. \\
\hline $\begin{array}{l}4-13 \text { wks. } \\
13-26 \text { wks. } \\
26-39 \text { wks. } \\
39 \text { wks. }-1 \text { yr. } \\
1 \text { yr. }-18 \text { mos. } \\
18 \text { mos. }-2 \text { yrs. } \\
2-3 \text { yrs. }\end{array}$ & $\begin{array}{l}76 \\
86 \\
80 \\
70 \\
70 \\
50 \\
25\end{array}$ & $\begin{array}{l}2.4 \\
1.6 \\
1.0 \\
0.7 \\
0.5 \\
0.5 \\
0.4\end{array}$ & $\begin{array}{l}6 \cdot 1 \\
4 \cdot 0 \\
2 \cdot 6 \\
1 \cdot 7 \\
1 \cdot 4 \\
1 \cdot 2 \\
1 \cdot 1\end{array}$ & $\begin{array}{l}65 \\
73 \\
68 \\
66 \\
66 \\
44 \\
24\end{array}$ & $\begin{array}{l}2.2 \\
1.7 \\
0.9 \\
0.7 \\
0.6 \\
0.5 \\
0.3\end{array}$ & $\begin{array}{l}5 \cdot 5 \\
4 \cdot 2 \\
2 \cdot 4 \\
1 \cdot 8 \\
1 \cdot 6 \\
1 \cdot 2 \\
0 \cdot 8\end{array}$ \\
\hline
\end{tabular}

MEAN 'ADJUSTED' INCREMENTS OF CROWN-RUMP

representing the distance between the crown of the head and the rump over a line connecting the ischial tuberosities. (See Bean, 1922; Knott, 1941; Falkner, 1957, etc.) Results are given in Tables 11 and 12.

Lower-segment length is obtained by simple subtraction of crown-rump length from total recumbent-length. The ratio of upper to lower segment is a useful index of growth and is commonly expressed as the percentage of the total length that is made up by the upper segment. It is known as the stem-stature index, and is expressed as:

\section{Stem-length (crown-rump length) $\times 100$ Recumbent-length (or height)}

The maximum standard deviation of the mean at any age in the Aberdeen or London children (where the raw data were available) was $\pm 1 \cdot 6$.

The index varies remarkably little between some widely different samples and decreases with age, as the lower limbs grow more rapidly than the upper segment. It reaches its final adult stable figure at approximately 12 years in the female and 16 years in the male. At the third month of foetal life it is of the order of 80.0 (Schultz, 1926), and at birth indexes from different racial groups vary little between 66.0 and 67.5 (examples: Japanese-Ito,

TABLE 13

MEAN STEM-STATURE INDEX OF CHILD STUDY CENTRE CHILDREN COMPARED WITH OTHER CHILDREN

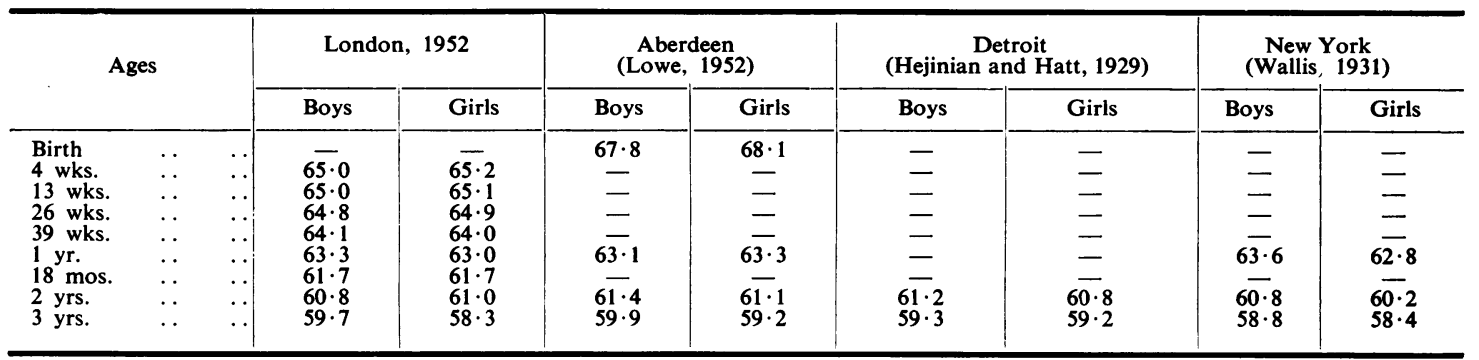

TABLE 14

MEAN HEAD CIRCUMFERENCES OF CHILD STUDY CENTRE CHILDREN AGED 4 WEEKS TO 3 YEARS

(In inches to nearest $0 \cdot 1 \mathrm{inch}$, and $\mathrm{cm}$. to nearest $\mathrm{mm}$.)

\begin{tabular}{|c|c|c|c|c|c|c|c|c|c|c|c|c|}
\hline \multirow{3}{*}{ Ages } & & & \multirow{3}{*}{ No. } & \multicolumn{4}{|c|}{ Boys } & \multicolumn{5}{|c|}{ Girls } \\
\hline & & & & \multicolumn{2}{|c|}{ Inches } & \multicolumn{2}{|c|}{$\mathrm{Cm}$. } & \multirow{2}{*}{ No. } & \multicolumn{2}{|c|}{ Inches } & \multicolumn{2}{|c|}{$\mathrm{Cm}$. } \\
\hline & & & & Mean & S.D. & Mean & S.D. & & Mean & S.D. & Mean & S.D. \\
\hline $\begin{array}{l}4 \text { wks. } \\
13 \text { wks. } \\
26 \text { wks. } \\
39 \text { wks. } \\
11 \text { yr. } \\
18 \text { mos. } \\
2 \text { yrs. } \\
3 \text { yrs. }\end{array}$ & $\begin{array}{l}. \\
\therefore \\
\therefore \\
\therefore \\
\therefore \\
\therefore\end{array}$ & $\begin{array}{l}. . \\
\cdots \\
. \\
. \\
. \\
. \\
.\end{array}$ & $\begin{array}{l}82 \\
96 \\
94 \\
83 \\
79 \\
83 \\
58 \\
35\end{array}$ & $\begin{array}{l}14 \cdot 7 \\
16 \cdot 0 \\
17 \cdot 3 \\
18 \cdot 0 \\
18 \cdot 4 \\
19.0 \\
19 \cdot 4 \\
19 \cdot 8\end{array}$ & $\begin{array}{l}0.4 \\
0.4 \\
0.5 \\
0.5 \\
0.5 \\
0.5 \\
0.6 \\
0.5\end{array}$ & $\begin{array}{l}37 \cdot 4 \\
40 \cdot 7 \\
43 \cdot 8 \\
45 \cdot 7 \\
46 \cdot 8 \\
48 \cdot 3 \\
49 \cdot 3 \\
50 \cdot 4\end{array}$ & $\begin{array}{l}1 \cdot 1 \\
1.1 \\
1.2 \\
1.3 \\
1.3 \\
1.3 \\
1.4 \\
1.3\end{array}$ & $\begin{array}{l}77 \\
84 \\
81 \\
78 \\
75 \\
74 \\
57 \\
32\end{array}$ & $\begin{array}{l}14 \cdot 4 \\
15 \cdot 6 \\
16 \cdot 8 \\
17 \cdot 6 \\
18 \cdot 0 \\
18 \cdot 5 \\
18 \cdot 8 \\
19 \cdot 5\end{array}$ & $\begin{array}{l}0.4 \\
0.5 \\
0.4 \\
0.5 \\
0.5 \\
0.4 \\
0.4 \\
0.4\end{array}$ & $\begin{array}{l}36 \cdot 5 \\
39 \cdot 7 \\
42 \cdot 7 \\
44 \cdot 6 \\
45 \cdot 7 \\
47 \cdot 0 \\
47 \cdot 9 \\
49 \cdot 5\end{array}$ & $\begin{array}{l}1 \cdot 0 \\
1 \cdot 2 \\
1 \cdot 1 \\
1 \cdot 2 \\
1 \cdot 2 \\
1 \cdot 1 \\
1 \cdot 1 \\
1 \cdot 1\end{array}$ \\
\hline
\end{tabular}


1936; American-Bakwin and Bakwin, 1934 ; Italian-Tatafiore, 1935). At 8 years it approximates 54.5 (Wallis, 1931). By puberty it is of the order of 51.5 to 52.0 in both sexes, and thereafter remains at this minimum amount. The index is influenced by age, sex, race and constitutional type. There are two areas of very low upper-segment length-Central Africa and Native Australasia; and two 'high' areas-Central Africa (Pygmies) and the Arctic (Bean, 1922); but otherwise the index appears remarkably stable and comparable in distribution.

Bean (1922) stated that in different samples the female index was generally larger than the male, regardless of constitutional type and stature. The present sample of children does not indicate that such differences appear in the first three years of life at least.

In certain diseases the index is altered and may give a useful additional diagnostic indication. As an example, in cretinism the dwarfism includes retardation of growth of the lower limbs, and this manifests itself in the index. Five cretins recently seen had the following indices: two aged 4 weeksmean of $69 \cdot 0$; two aged 3 months-mean of $68 \cdot 1$; and one aged 6 months $-66 \cdot 9$. By comparison with Table 13 it will be seen that these indices are high.

Head Circumference. This is taken with a flexible, narrow-width steel tape and represents the maximum circumference, with the tape passing round the occipital and frontal areas and held perpendicular to the mid-sagittal plane.

TABLE 15

'ADJUSTED' MEAN INCREMENTS OF HEAD CIRCUMFERENCE IN 13-WEEK PERIODS OF CHILD STUDY CENTRE CHILDREN AGED 4 WEEKS TO 3 YEARS

(In inches to nearest $\mathbf{0 . 1}$ inch, and $\mathrm{cm}$. to nearest $\mathrm{mm}$.)

\begin{tabular}{|c|c|c|c|c|c|c|}
\hline \multirow{3}{*}{ Ages } & \multicolumn{3}{|c|}{ Boys } & \multicolumn{3}{|c|}{ Girls } \\
\hline & \multirow{2}{*}{ No. } & \multicolumn{2}{|c|}{$\begin{array}{l}\text { ‘Adjusted’ Mean } \\
\text { Increment }\end{array}$} & \multirow{2}{*}{ No. } & \multicolumn{2}{|c|}{$\begin{array}{l}\text { ‘Adjusted’ Mean } \\
\text { Increment }\end{array}$} \\
\hline & & Inches & $\mathrm{Cm}$. & & Inches & . Cm. \\
\hline $\begin{array}{l}\text { 4-13 wks. } \\
13-26 \text { wks. } \\
26-39 \text { wks. } \\
39 \text { wks.-1 yr. } \\
1 \text { yr.-18 mos. } \\
18 \text { mos.-2 yrs. } \\
2-3 \text { yrs. }\end{array}$ & $\begin{array}{l}77 \\
87 \\
79 \\
69 \\
67 \\
49 \\
33\end{array}$ & $\begin{array}{l}1 \cdot 9 \\
1 \cdot 2 \\
0.8 \\
0 \cdot 5 \\
0 \cdot 3 \\
0 \cdot 2 \\
0 \cdot 1\end{array}$ & $\begin{array}{l}4 \cdot 8 \\
3 \cdot 1 \\
2 \cdot 0 \\
1 \cdot 2 \\
0 \cdot 7 \\
0 \cdot 5 \\
0 \cdot 3\end{array}$ & $\begin{array}{l}65 \\
73 \\
69 \\
67 \\
62 \\
26 \\
28\end{array}$ & $\begin{array}{l}1.9 \\
1.2 \\
0.7 \\
0.4 \\
0.3 \\
0.2 \\
0.1\end{array}$ & $\begin{array}{l}4 \cdot 8 \\
3 \cdot 1 \\
1 \cdot 9 \\
1 \cdot 1 \\
0 \cdot 7 \\
0 \cdot 5 \\
0 \cdot 4\end{array}$ \\
\hline
\end{tabular}

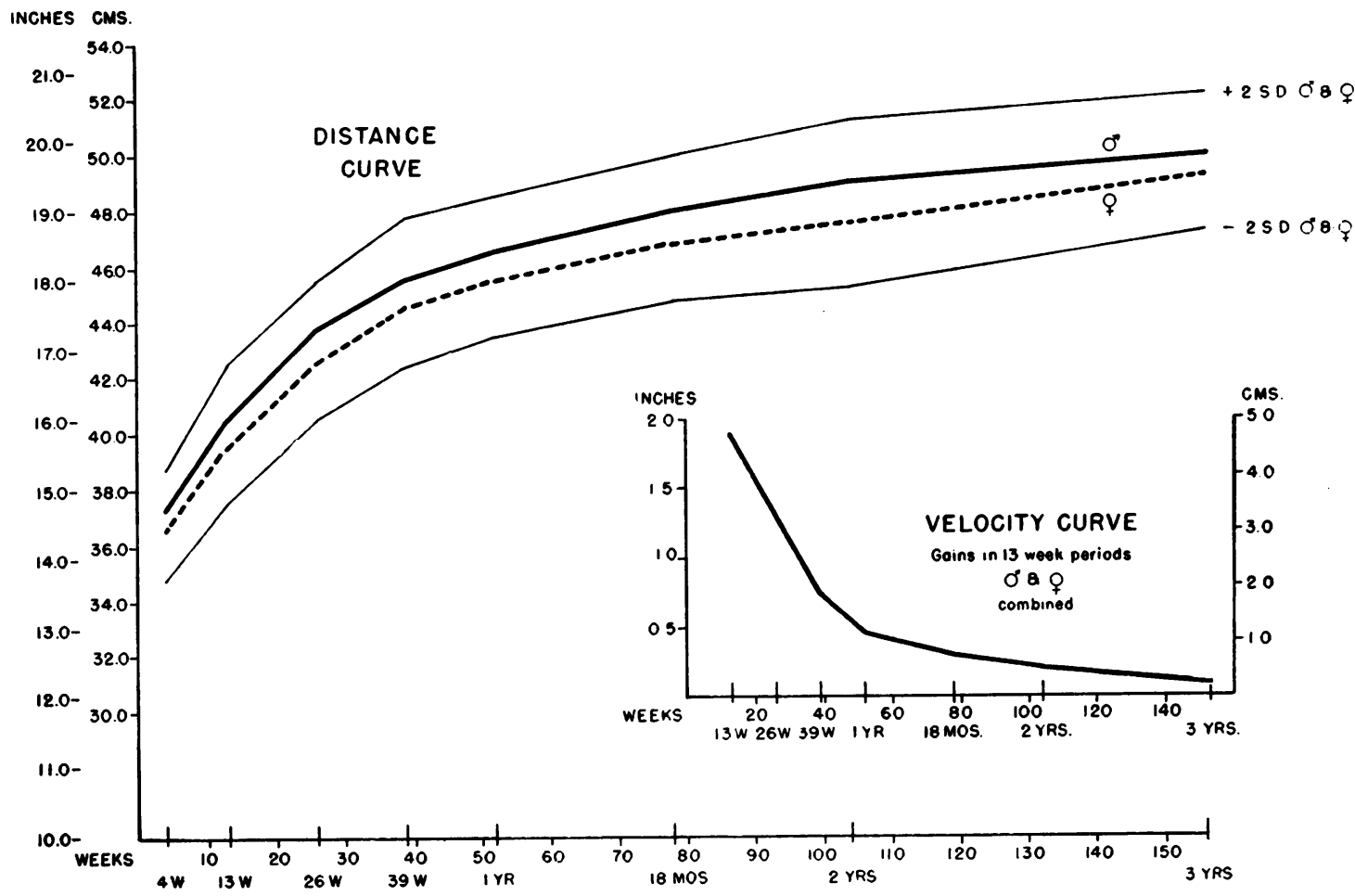

FIG. 3.-Head circumferences: distance and velocity curves. In the distance curves, for simplicity, boys and girls are combined to give the 2 S.D. curves. Boys and girls are combined to give the velocity curves, since their increments are almost identical. 
Head circumference is a useful measurement clinically in early childhood, particularly when hydrocephalus, subdural haematoma or neoplasms are in question. Correlations between head measurement, cranial capacity and intelligence are, however, uniformly low and of the order of 0.12 (Broom, 1932; Paterson, 1930). Incremental data may be of most use. Although the boys' mean head circumferences are consistently larger than the girls', the mean increments are practically identical.

Fig. 3 shows graphically* the data from Tables 14 and 15. The incremental curve is of boys and girls combined.

\section{Summary}

Means and mean increments of recumbent-length, weight, crown-rump length and head circumference are given for children from a longitudinal developmental study in London. The children were born in 1952-4. Standard deviations and percentiles are given. Comparative lengths and weights, and increments of both, are given for samples of children born from 1923 to 1954 . The secular trend for children to become larger continues. The relationship between early, late and adult life is discussed. There is very little relationship between size at 4 weeks and 1 year, but a good relationship between 1 year and 2 years. Size appears independent of rate of growth, which shows little relationship from any age to any age.

* See footnote on page 2 .
Stem-stature indices are given and comparisons with other samples made. Their usefulness is discussed.

I am much indebted and grateful to Professor Alan Moncrieff for constant encouragement and guidance; to Dr. W. M. Krogman, who read and criticized this paper; and to Miss Coral Tibbetts, Mr. Richard Westland and Mrs. Nancy Singleton for computational and secretarial assistance.

\section{REFERENCES}

Acheson, R. M., Kemp, F. H. and Parfit, J. (1955). Lancet, 1, 691. Annotation (1955). Ibid., 2, 1327.

Bakwin, H. and Bakwin, R. M. (1934). Hum. Biol., 6, 612

Bean, R. B. (1922). Amer. J. phys. Anthrop., 5, 349.

Boyd, E. (1929). Ibid., 13, 389.

Broom, M. E. (1932). School and Society, 36, 703.

Falkner, F. (1957). Ed. Internationally Cooperative Research in Ċhild Development. Centre Int. de l'Enfance, Paris.

Gore, A. T. and Palmer, W. T. (1949). Lancet, 1, 385.

Gray, H. and Ayres, J. G. (1931). Growth in Private School Children. University of Chicago Press

Hindley, C. B. (1953). Lancet, $2,142$.

Ito, P. K. (1936). Amer. J. Dis. Child., 52, 321.

Hejinian, L. and Hatt, E. (1929). Amer. J. phys. Anthrop., 13, 287.

Knott, V. B. (1941). Univ. Iowa Studies in Child Welfare, 18, No. 3. [1948].

Krogman, W. M. (1950). Monogr. Soc. Res. Child Dev., 13, No. 3. (1956). Ibid., 20, No. 1. [1955].

Lincoln, E. A. (1930). J. genet. Psychol., 38, 445.

Low, A. (1952). Growth of Children. Univ. of Aberdeen.

Moore, T., Hindley, C. B. and Falkner, F. (1954). Brit. med. J., 2, , 1132

Myers, B. (1926). Brit. J. Child. Dis., 23, 87.

Palmer, C. E. (1930). Anat. Rec., 45, 234

Paterson, D. G. (1930). Physique and Intellect. New York

Redfield, J. E. and Meredith, H. V. (1938). Child Developm., 9, 293

Schultz, A. H. (1926). Quart. Rev. Biol., 1, 465.

Simmons, K. (1944). Monogr. Soc. Res. Child Dev., 9, No. 1.

Simmons, K. (1944). Monogr. Soc. Res.

Tanner, J. M. (1951). Hum. Biol., 23, 93 .

(1955), Growth at Adolescence. Oxford.
Healy, M. J. R., Lockhart, R. D., MacKenzie, J. D., and Whitehouse, R. H., (1956). Arch.' Dis. Childh., 31, 372.

Tatafiore, E. (1935). Pediatria (Napoli), 43, 422.

Thomson, J. (1956). Arch. Dis Childh 31, 382.

Wallis, R. S. (1931). Univ. Iowa. Studies in Child Welfare, Vol. 5. No. 1 . 